\title{
Current-Driven Domain-Wall Depinning in Pt/CoFe/Pt Nanowires with Perpendicular Magnetic Anisotropy
}

\author{
Kab-Jin Kim, Jae-Chul Lee, and Sug-Bong Choe* \\ Department of Physics, Seoul National University, Seoul 151-742, Korea
}

(Received 12 June 2009, Received in final form 24 July 2009, Accepted 4 August 2009)

\begin{abstract}
The spin transfer torque efficiency was determined experimentally by observing the current-driven domainwall depinning of $\mathrm{Pt} / \mathrm{CoFe} / \mathrm{Pt}$ nanowires with perpendicular magnetic anisotropy. The depinning time was exponentially proportional to the applied magnetic field, and was well explained by the Néel-Brown formula. The depinning time and threshold magnetic field were varied considerably by injecting current into the nanowire. The spin transfer torque efficiency was estimated to be $(7.2 \pm 0.9) \times 10^{-15} \mathrm{Tm}^{2} / \mathrm{A}$ from the linear dependence of the threshold current density with respect to the applied magnetic field.
\end{abstract}

Keywords : spin transfer torque, domain wall motion, perpendicular magnetic anisotropy

\section{Introduction}

Current-driven magnetic domain-wall motion, which was first predicted by Berger [1], has been studied extensively for the possibility of the next generation logic and memory devices [2, 3]. Many theoretical and experimental approaches have been attempted to better understand the mechanism [2-7]. In studies of Permalloy nanowires with inplane magnetic anisotropy, it was verified that multiple domain walls are successfully moved by the spin polarized current via the spin angular momentum transfer [3-5]. However, since the domain wall width is large $(>100 \mathrm{~nm})$ in Permalloy nanowires, the spin transfer torque phenomenon is governed mainly by an adiabatic process [6]. In addition, such a wide domain wall has a complicated two-dimensional spin structure, which prevents a direct comparison with the one-dimensional collective model due to the occurrence of the domain wall deformation during current pulse injection [7].

Quite recently, a nanowire structure with perpendicular magnetic anisotropy was introduced as another candidate for current driven domain wall motion [8-16]. The onedimensional collective model was used to explain the domain wall behavior because nanowires with perpendicular magnetic anisotropy exhibit simple domain wall structures of either Bloch or Néel domain walls with a narrower

*Corresponding author: Tel: +82-2-880-9254

Fax: +82-2-884-9254, e-mail: sugbong@snu.ac.kr wall width [8]. The narrow domain wall induces a larger magnetization gradient, which generates a large nonadiabatic torque [6]. Larger nonadiabaticity results in higher efficient of the current-driven domain wall motion [9], [10]. This paper reports an experimental estimation of the spin transfer torque efficiency of $\mathrm{Pt} / \mathrm{CoFe} / \mathrm{Pt}$ nanowires, based on the observation of a current-driven domain-wall depinning phenomena.

\section{Experimental Procedure}

In this study, $50-\AA \mathrm{Ta} / 25-\AA \mathrm{Pt} / 10-\AA \mathrm{Co}_{90} \mathrm{Fe}_{10} / 10-\AA \mathrm{Pt}$ films were deposited on a $\mathrm{Si} / \mathrm{SiO}_{2}(1000 \AA)$ substrate by dc-magnetron sputtering in an ultrahigh vacuum environment. Using an alternating gradient magnetometer, the saturation magnetization $M_{\mathrm{S}}$ and coercive field $H_{\mathrm{C}}$ were measured to be $9.86 \times 10^{5} \mathrm{~A} / \mathrm{m}$ and $12.5 \mathrm{mT}$, respectively, as shown in Fig. 1(a). 10- $\mu \mathrm{m}$-long and $160-n m$-wide wires were then fabricated by electron-beam lithography and ion milling onto the film. A diamond shaped pad was introduced to examine the domain wall depinning at the neck between the nanowire and pad. Fig. 1(b) and (c) show the sample geometry and a typical hysteresis loop of the nanowire, respectively.

The current-driven domain-wall depinning time was measured using a scanning magneto-optical Kerr effect microscope [17]. The nanowire was first saturated under a sufficiently high magnetic field and the domain wall was formed at the neck between the nanowire and pad via a 


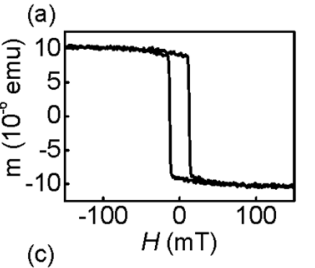

(b)

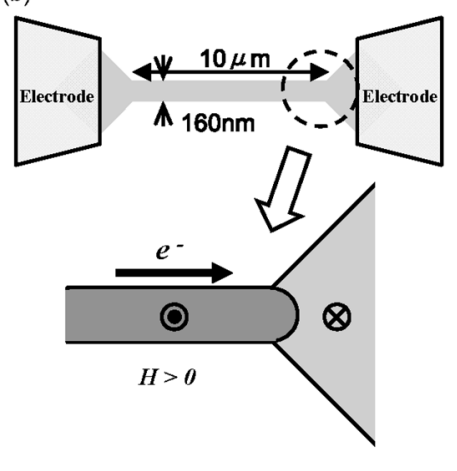

Fig. 1. (a) Out-of-plane hysteresis loop of the film measured using an alternating gradient magnetometer. (b) The sample structure and measurement geometry. (c) Typical out-of-plane hysteresis loop of nanowires measured using a magneto-optical Kerr effect microscope.

thermo-magnetic writing process. Thermo-magnetic writing was carried out by focusing a high power laser $(7.5 \mathrm{~mW}$, $300 \mathrm{~ns})$ onto a small spot ( $\sim 500 \mathrm{~nm})$ under a weak reversed field $\left(H<0.5 H_{\mathrm{C}}\right)$. The domain wall depinning time after applying the magnetic field or spin polarized current was then monitored at the pad. An abrupt decrease in the magneto-optical Kerr effect signal was detected when the domain wall was depinned from the neck.

\section{Results and Discussion}

Fig. 2 shows the domain wall depinning time with respect to the applied magnetic field at zero current (open circle). The depinning time $\tau_{\mathrm{d}}$ is exponentially dependent on the applied field. The exponential dependence can be explained by a thermally activated magnetization process, which was predicted by Néel and Brown [18], as follows:

$$
\tau(H, J=0)=\tau_{0} \exp \left[\frac{M_{\mathrm{S}} V_{\mathrm{A}}}{k_{\mathrm{B}} T}\left(H_{\mathrm{d}}-H\right)\right],
$$

where $\tau_{0}$ is the inverse of the attempt frequency, $V_{\mathrm{A}}$ is the activation volume, $k_{\mathrm{B}} T$ is the thermal fluctuation energy, and $H_{\mathrm{d}}$ is the depinning field at $0 \mathrm{~K}$. From the best linear fit with Eq. (1), the depinning field $H_{\mathrm{d}}$ and activation volume $V_{\mathrm{A}}$ were determined to be $45.2 \pm 4.8 \mathrm{mT}$ and (4.21 $\pm 0.27) \times 10^{-24} \mathrm{~m}^{3}$, respectively, by assuming a typical value of $\tau_{0} \sim 1 \mathrm{~ns}$. In general, the depinning field $H_{\mathrm{d}}$ is the composed of two contributions, the intrinsic depinning field $H_{\mathrm{d}}^{\text {int }}$ due to the grain boundaries of virgin films and the extrinsic depinning field $H_{\mathrm{d}}^{\text {ext }}$ from the geometric constriction of the nanodevice structure. The extrinsic depinning field is given by $\sigma_{\mathrm{W}} \sin \theta / 2 M_{\mathrm{S}}\left(h+1 / 2 l_{\mathrm{W}} \sin \theta\right)$ [19], where, $\sigma_{\mathrm{W}}$ is the domain wall energy density per unit area, $\theta$ is the angle of the pad slope, and $l_{\mathrm{W}}$ is the

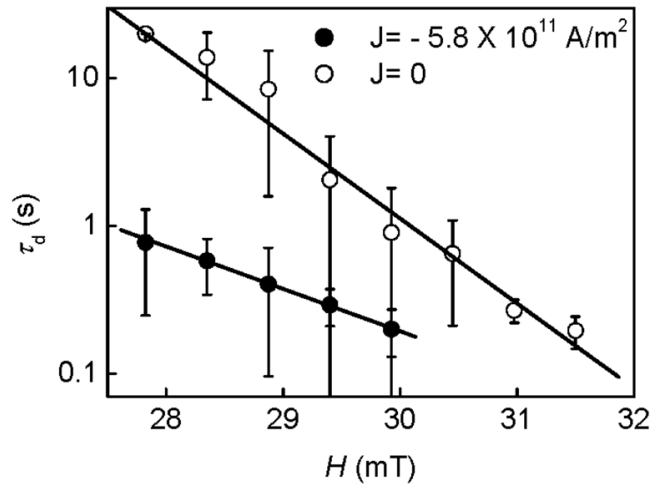

Fig. 2. The domain wall depinning time with respect to the magnetic field strength for (a) $J=0$ (open circle) and (b) $J=$ $-5.8 \times 10^{11} \mathrm{~A} / \mathrm{m}^{2}$ (solid circle), respectively. Each data point is an average of 5 measurements, and the error bar indicates the standard deviation. The solid lines show the lines of best fit with Eq. (1).

domain wall width. Using the typical values for our system, $H_{\mathrm{d}}^{\text {ext }}=38.0 \mathrm{mT}$ giving an intrinsic depinning field of approximately $H_{\mathrm{d}}^{\mathrm{int}}=7.2 \mathrm{mT}$. The magnitude of the intrinsic depinning field was confirmed to be consistent with the propagation field of the films.

To study the effect of the current on the domain wall depinning phenomena, a constant dc current was applied to the nanowire and a field-dependent depinning time was measured. The solid circular symbols in Fig. 2 show the depinning time with respect to the magnetic field under a negative dc current, $-5.8 \times 10^{11} \mathrm{~A} / \mathrm{m}^{2}$. Current injection affects the depinning time of the domain wall. The positive dc current did not alter the depinning time.

The depinning time was measured as a function of the magnitude of the current under a fixed external magnetic field to observe the effect from the current injection. The results are shown in Fig. 3. The depinning time again

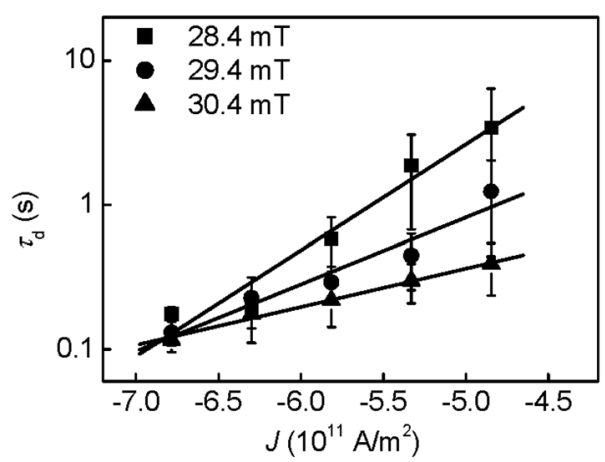

Fig. 3. The domain wall depinning time with respect to the current density for several magnetic field strengths, as denoted in the figure. Each data point is an average of 5 measurements, and the error bar indicates the standard deviation in both figures. The solid line shows the line of best fit with Eq. (2). 


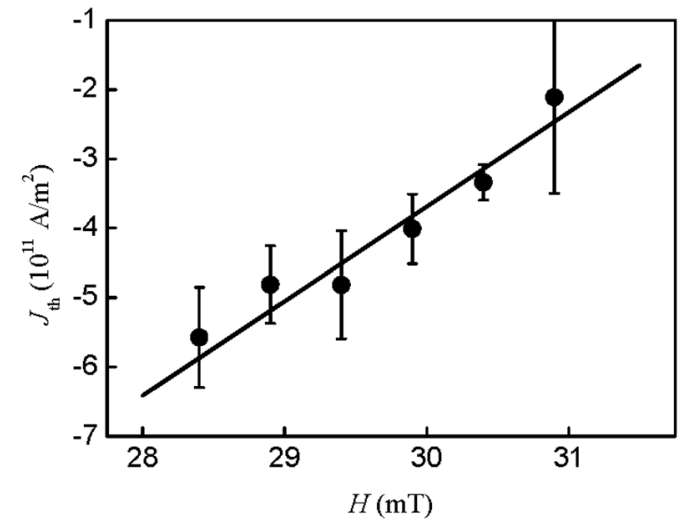

Fig. 4. The threshold current density with respect to the external magnetic field. The solid line shows the line of best fit.

exhibits the exponential dependence on the current density. To explain the exponential behavior, the thermally activated magnetization process was extended to [9]

$$
\tau(H, J)=\tau_{0} \exp \left[\frac{M_{\mathrm{S}} V_{\mathrm{A}}}{k_{\mathrm{B}} T}\left(H_{\mathrm{d}}+\varepsilon J-H\right)\right],
$$

where $\varepsilon$ is the spin transfer torque efficiency, which corresponds to the proportional coefficient between the current density and effective magnetic field i.e. $\varepsilon=\Delta H_{\text {eff }} / \Delta J$ [11].

The spin transfer torque efficiency was finally estimated by measuring the threshold current density with respect to the external magnetic field. Here, the threshold current density is defined as the current density for domain wall depinning at a depinning time of $\tau=1 \mathrm{~s}$ [9], which was obtained by fitting the exponential dependence in Fig. 3, as shown in Fig. 4. The threshold current density was plotted with respect to the external magnetic field (Fig. 4). The threshold current density was linearly dependent on the external magnetic field. The linearity was expressed by the best fit as $J(H)=J_{0}\left[1-H / H_{0}\right]$, where the threshold current density at zero magnetic field, $J_{0}=-(4.5$ $\pm 0.4) \times 10^{12} \mathrm{~A} / \mathrm{m}^{2}$, and the zero-current depinning field, $H_{0}$ $=(3.25 \pm 0.03) \times 10^{-2} \mathrm{~T}$, respectively. The spin transfer torque efficiency was then obtained from the equation $\varepsilon=$ $\left|H_{0} / J_{0}\right|$, as $(7.2 \pm 0.6) \times 10^{-15} \mathrm{Tm}^{2} / \mathrm{A}$. This value is smaller than that obtained for $\mathrm{Co} / \mathrm{Pt}$ nanowires [11] but similar to that of permalloy nanowires [20].

\section{Conclusion}

Current-driven domain-wall depinning was examined in ferromagnetic $\mathrm{Pt} / \mathrm{CoFe} / \mathrm{Pt}$ nanowires with perpendicular magnetic anisotropy. The spin transfer torque efficiency was estimated to be $(7.2 \pm 0.6) \times 10^{-15} \mathrm{Tm}^{2} / \mathrm{A}$ from the depinning time measurements at different magnetic fields and/or the currents.

\section{Acknowledgement}

This study was supported by the KOSEF through the NRL program (R0A-2007-000-20032-0). The authors wish to thank Sunae Seo, Chang-Won Lee, and Young-Jin Cho at Samsung Advanced Institute of Technology for preparing the films and Kyung-Ho Shin at Korea Institute of Science and Technology for patterning the nanostructures.

\section{References}

[1] L. Berger, J. Appl. Phys. 55, 1954 (1984).

[2] D. A. Allwood, G. Xiong, C. C. Faulkner, D. Atkinson, D. Petit, and R. P. Cowburn, Science 309, 1688 (2005).

[3] S. S. P. Parkin, M. Hayashi, and L. Thomas, Science 320, 190 (2008).

[4] M. Hayashi, L. Thomas, R. Moriya, C. Rettner, and S. S. P. Parkin, Science 320, 209 (2008).

[5] M. Hayashi, L. Thomas, C. Rettner, R. Moriya, Y. B. Bazaliy, and S. S. P. Parkin, Phys. Rev. Lett. 98, 037204 (2007).

[6] G. Tatara and H. Kohno, Phys. Rev. Lett. 92, 086601 (2004).

[7] M. Kläui, P.-O. Jubert, R. Allenspach, A. Bischof, J. A. C. Bland, G. Faini, U. Rüdiger, C. A. F. Vaz, L. Vila, and C. Vouille, Phys. Rev. Lett. 95, 026601 (2005).

[8] S.-B. Choe, Appl. Phys. Lett. 92, 062506 (2008).

[9] D. Ravelosona, D. Lacour, J. A. Katine, B. D. Terris, and C. Chappert, Phys. Rev. Lett. 95, 117203 (2005).

[10] M. Feigenson, J. W. Reiner, and L. Klein, Phys. Rev. Lett. 98, 247204 (2007).

[11] O. Boulle, J. Kimling, P. Warnicke, M. Kläui, U. Rüdiger, G. Malinowski, H. J. M. Swagten, B. Koopmans, C. Ulysse, and G. Faini, Phys. Rev. Lett. 101, 216601 (2008).

[12] S.-W. Jung, W. Kim, T.-D. Lee, K.-J. Lee, and H.-W. Lee, Appl. Phys. Lett. 92, 202508 (2008).

[13] S.-W. Jung and H.-W. Lee, J. Magnetics 12, 1 (2007).

[14] H. Tanigawa, K. Kondou, T. Koyama, K. Nakano, S. Kasai, N. Ohshima, S. Fukami, N. Ishiwata, and T. Ono, Appl. Phys. Express 1, 011301 (2008).

[15] T. Koyama, G. Yamada, H. Tanigawa, S. Kasai, N. Ohshima, S. Fukami, N. Ishiwata, Y. Nakatani, and T. Ono, Appl. Phys. Express 1, 101303 (2008).

[16] T. A. Moore, I. M. Miron, G. Gaudin, G. Serret, S. Auffret, B. Rodmacq, A. Schuhl, S. Pizzini, J. Vogel, and M. Bonfim, Appl. Phys. Lett. 93, 262504 (2008).

[17] K.-J. Kim, J.-C. Lee, S.-M. Ahn, K.-S. Lee, C.-W. Lee, Y. J. Cho, S. Seo, K.-H. Shin, S.-B. Choe, and H.-W. Lee, Nature 458, 740 (2009).

[18] S.-B. Choe, D.-H. Kim, K.-S. Ryu, H.-S. Lee, S.-C. Shin, J. Appl. Phys. 99, 103902 (2006).

[19] K.-J. Kim and S.-B. Choe, J. Magn. Magn. Mater. 321, 2197 (2009).

[20] M. Laufenberg, W. Bührer, D. Bedau, P.-E. Melchy, M. Kläui, L. Vila, G. Faini, C. A. F. Vaz, J. A. C. Bland, and U. Rüdiger, Phys. Rev. Lett. 97, 046602 (2006). 